2009;9:389-400. https://doi.org/10.1089/vbz.2008.0160

3. Vazquez-Prokopec GM, Chaves LF, Ritchie SA, Davis J, Kitron U. Unforeseen costs of cutting mosquito surveillance budgets. PLoS Negl Trop Dis. 2010;4:e858. https:/ / doi.org/10.1371/journal.pntd.0000858

4. Jordan RA, Schulze TL, Jahn MB. Effects of reduced deer density on the abundance of Ixodes scapularis (Acari: Ixodidae) and Lyme disease incidence in a northern New Jersey endemic area. J Med Entomol. 2007;44:752-7. https://doi.org/10.1093/jmedent/44.5.752

5. Schulze TL, Jordan RA, Schulze CJ, Healy SP, Jahn MB, Piesman J. Integrated use of 4-Poster passive topical treatment devices for deer, targeted acaricide applications, and Maxforce TMS bait boxes to rapidly suppress populations of Ixodes scapularis (Acari: Ixodidae) in a residential landscape. J Med Entomol. 2007;44:830-9. https://doi.org/10.1093/jmedent/44.5.830

Address for correspondence: Robert A. Jordan, Tick-borne Disease Program, Monmouth County Mosquito Control Division, 1901 Wayside Rd, Tinton Falls, New Jersey, USA; email: Robert.Jordan@co.monmouth.nj.us

\section{Rhabdomyolysis as Potential Late Complication Associated with COVID-19}

\author{
Kok Hoe Chan, Jihad Slim \\ Author affiliation: Saint Michael's Medical Center, New York \\ Medical College, Newark, New Jersey, USA
}

DOI: https://doi.org/10.3201/eid2610.202225

To the Editor: Jin and Tong described a patient with severe coronavirus disease (COVID-19) in whom rhabdomyolysis developed on day 9 of hospitalization (1). The interplay between severe acute respiratory syndrome coronavirus 2 and rhabdomyolysis is not yet understood; we consider possible etiologies for this case of rhabdomyolysis.
We reported 2 case-patients with COVID-19 who also had weakness and elevated creatinine kinase levels (but no respiratory symptoms) (2). As part of his COVID-19 treatment regimen, the patient reported by Jin and Tong received lopinavir and meropenem, which can cause rhabdomyolysis $(3,4)$. Meropenem is associated with rhabdomyolysis by inducing severe hypomagnesemia and hypokalemia; it would be helpful to know the trends in the patient's electrolytes before rhabdomyolysis developed (3). A cytokine storm might also have caused this complication because rhabdomyolysis developed on day 15 of COVID-19 symptoms and coincided with the peak of inflammatory markers (C-reactive protein). On the other hand, the combination of hypoxia and hypercoagulability might have induced an ischemic event that inhibited blood flow to the involved muscles, triggering rhabdomyolysis.

Clinicians treating rhabdomyolysis concurrent with COVID-19 must assess the many differential diagnoses, including severe acute respiratory syndrome coronavirus 2-induced myositis, reactions to medication, cytokine storm, hypoxia, or a thromboembolic event. This differential diagnosis is crucial because each condition has a distinct therapeutic approach.

\section{References}

1. Jin $\mathrm{M}$, Tong $\mathrm{Q}$. Rhabdomyolysis as potential late complication associated with COVID-19. Emerg Infect Dis. 2020;26:1618-20. https:// doi.org/10.3201/ eid2607.200445

2. Chan KH, Farouji I, Abu Hanoud A, Slim J. Weakness and elevated creatinine kinase as the initial presentation of coronavirus disease 2019 (COVID-19). Am J Emerg Med. 2020 May 11 [Epub ahead of print].

3. de Kanter CT, Keuter M, van der Lee MJ, Koopmans PP, Burger DM. Rhabdomyolysis in an HIV-infected patient with impaired renal function concomitantly treated with rosuvastatin and lopinavir/ritonavir. Antivir Ther. 2011;16:435-7. https://doi.org/10.3851/IMP1747

4. Margolin L. Impaired rehabilitation secondary to muscle weakness induced by meropenem. Clin Drug Investig. 2004;24:61-2. https:/ / doi.org/10.2165/00044011-20042401000008

Address for correspondence: Kok Hoe Chan, Saint Michael's Medical Center, Newark, NJ 07101, USA; email: kchan2@ primehealthcare.com 